\title{
A Novel approach of Modeling and Simulation of Analog Modulation Techniques Using Simulink
}

\author{
R. Prakash Kumar \\ CVR College of Engineering/ECE Dept, Hyderabad, India. \\ Email:Prakash.rachmagdu@gmail.com
}

\begin{abstract}
This paper presents step by step procedure for designing analog modulation techniques such as Amplitude Modulation (AM) and Frequency Modulation (FM).These techniques are simulated in Simulink environment. AM and FM broadcasting range is of $550-1650 \mathrm{KHz}$ and $88-108 \mathrm{MHz}$ respectively. Tuning of these frequencies is difficult in implementation. To implement these modulation techniques, MATLAB and Simulink tool has been used. These tools will help the engineers for simulating a complete system before embarking on actual prototype. Simulation results show the capability of adequate system performance. In this paper AM and FM systems are simulated using MATLAB and Simulink and their performance is presented.
\end{abstract}

Index Terms: AM, FM and Demodulators.

\section{INTRODUCTION}

Dynamic system can be simulated, modeled and analyzed by simulation software. Simulink software is used in this paper. It provides graphical user interface (GUI) and block diagram model design just by click and drag it, also simulates both the linear and non-linear system performance modeled in continuous, discrete and hybrid. It supports multirate simulation model. It supports not only the library blocks but also provides customize and create the user defined blocks. These models are simulated either in Simulink or MATLAB command prompt. Interactive work is convenient by using menus in Simulink, where as Batch simulations are running and useful in command-line approach. In Simulink results of simulation will seen even when simulation is running and parameters are change immediately. These results not only seen on display blocks and scopes but also can be used for visualization and post processing for MATLAB workspace. Simulink and MATLAB's are integrated. In integrated environment the models can be analyzed, simulated and revised at any point in Communication Radio environment. Conveying the information over varying the carrier wave frequency then it is called Frequency Modulation (FM). Similarly conveying the information over varying the carrier wave amplitude then it is called Amplitude Modulation (AM).In radio communication environment transmit the data /voice without disturbance, but communication system suffers from disturbances like interference and noise. In FM, [1] the instantaneous carrier frequency is proportional to the amplitude of input signal, where as in digital the carrier frequency is varied among set of discrete values is known as FSK (Frequency Shift Keying). In today's, world the most prevalent form of communication media is the FM radio.
FM radio products and capability increases by portable devices like mobile phones and MP3 players because listeners in the entire world can continue to buy a portable FM radio. It is a challenging task to the design engineers to design, understanding and creating the high performance standalone radio products in next generation for multi use devices. The CCITT (Consultative Committee for International Telephony and Telegraphy) has allocated the FM station frequencies from 88 to $108 \mathrm{MHz}$, each station is designed to $200 \mathrm{KHz}$ to transmit the voice signal similarly AM stations are designed 550 to $1650 \mathrm{KHz}$,each station has $10 \mathrm{KHz}$ bandwidth[6].These modulations schemes are used for transmission of television signal. It is quite demand task for building the channels in communication radio environment, because it contains both audio and picture signal.

Buying this real network devices and system development is very costly. An alternative and better choice has become computer simulation package. These simulations are not only building the model. These models are designed from physical layer to network layer establishment. System simulators enable researchers, network administrators, and other interested users to test new network technologies and protocols before using them on real networks. The real models consist of set mathematical formulae for building the real phenomenon by using the software simulation. It is frequently used in equipment design and close to the design specifications without any expensive modification process [7]. Software simulation has proven to be a powerful tool in modeling a complete communication channel before embarking on the real prototype. In recent years simulations can be done for even complex systems like FDMA (Frequency Division Multiple Access), CDMA (Code Division Multiple Access) and OFDMA (Orthogonal Frequency Division Multiple Access) .These models can be used for not only understanding the basic structure but also evolution of variety design principles and computer (system) simulations are involved. Based on this, computer simulation sub-fields have been proposed, as well as a large number of tools and applications having been designed and developed. The benefits of computer simulation are becoming more generally recognized in several domains and areas. Some of the computer software packages usually used by Engineers for analysis and simulation of physical systems such as MATLAB/SIMULINK, RMxprt/Maxwell2D/3D, SIMPLORER, HFSSV10, FDTD/ Varipose/XGTD, COMSOL,5SPICE, ANSYS, 
Electronic Workbench etc. Using these tools researchers are more intense in problem solving experience[8],but today's the availability of the software packages is now reshaping the role and applications of computer laboratory projects. User programs also provide an opportunity to easily conduct numerical experiments and to tackle realistic and more complicated problems. In this paper the amplitude and frequency Modulated radio station, modeled and simulated it using MATLAB and SIMULINK tools to predict adequately its performance and behavior.

\section{Modeling And Simulation Concepts}

The mathematical models, graphical, conceptual and abstract generation using modeling process. The collection of methods, theory and techniques about modeling specialization is grown by science every day. Based on this assumption simulation can be defined as model can be created by using abstract representation of an existing or proposed system and what are the factors effecting the system performance and predict the performance of the system by using future behavior of the system by quantitatively described using equations and/or rules can be simulated[6]. As CSG (Computer Simulation Gaines) is [9] stated the number of good reasons for using computer simulation as a problem-solving tool. The main reasons are shown in the following list.

1. The physical system is not available

2. The experiment may be dangerous under danger of injury/damage or death/destruction

3. The cost of experimentation is too high

4. The experimenter does not compatible with the time constants

5. Control variables, and/or system parameters may be inaccessible.

Different Stages in simulation process is shown in fig1.

$$
\text { It consists of following stages }
$$

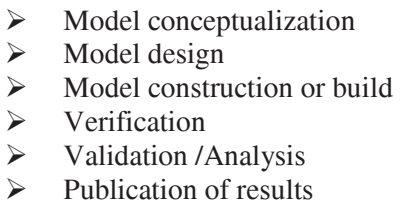

Replication

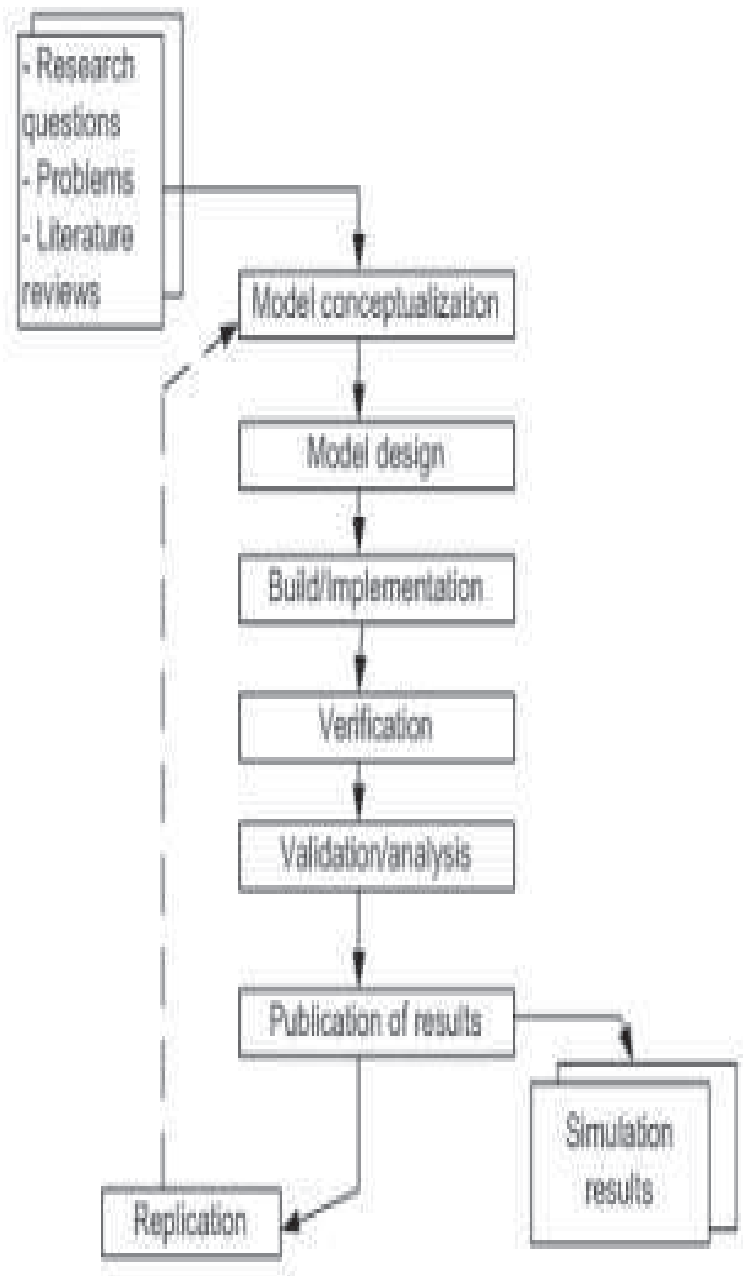

Fig. 1: A generic stages in the simulation

\section{Simulation Models \& Results}

A. Amplitude Modulation: Amplitude Modulation is defined as the Carrier wave amplitude is varied in accordance with the amplitude of modulating wave keeping as frequency and phase is constant. The standard form of amplitude modulated (AM) wave is defined equation (1)

$$
S(t)=A_{c} \cos \left(2 \pi f_{c} t\right)+k_{c} \frac{A_{r} A_{m}}{2}\left\{\cos \left(2 \pi\left(f_{c}+f_{m}\right) t\right)+\cos \left(2 \pi\left(f_{c}-f_{m}\right) t\right)\right\}
$$

Where $\mathrm{Ka}$ is a constant called amplitude sensitivity of the modulator. $f_{c}$ is the carrier frequency, $f_{m}$ is a modulating or message frequency, $\left(\mathrm{f}_{\mathrm{c}}+\mathrm{f}_{\mathrm{m}}\right)$ is upper side band frequency, $\left(\mathrm{f}_{\mathrm{c}}-\mathrm{f}_{\mathrm{m}}\right)$ is a lower side band frequency and $\mu$ is called modulation index.

Depending on the modulation index AM is classified into
(i).Under Modulation $(\mu<1)$
(ii). Exact Modulation $(\mu=1)$
(iii). Over Modulation $(\mu>1)$

The Simulink model of DSB-SC (Double Side Band Superseded Carrier) Modulation is shown in fig.2. one of 
the form of AM is DSB-SC .In this modulation $\mu$ value is greater than 1 .the main advantage of AM over DSB-SC is saving of transmission power. It also transmits only two side band frequencies and carrier frequency is suppressed.

DSB-SC modulated wave can be generated by using product modulator. The principle in this modulator is multiplication modulating and carrier signal. All these blocks are selected form the Simulink Library .The block properties are changed by right clicking on that block. This model is simulated with a modulating frequency of $1 \mathrm{~Hz}$ and carrier frequency of $10 \mathrm{~Hz}$ the modulated signal is shown in fig.3.these modulated signal is demodulated by using the synchronous detector. The principle in this detector is same carrier is multiplied in the receiver sections shown in fig.2. Resulting demodulated signal is passed through low pass filter signal shown in Fig.3. In this paper the filter used as analog butter worth filter order of 2 .

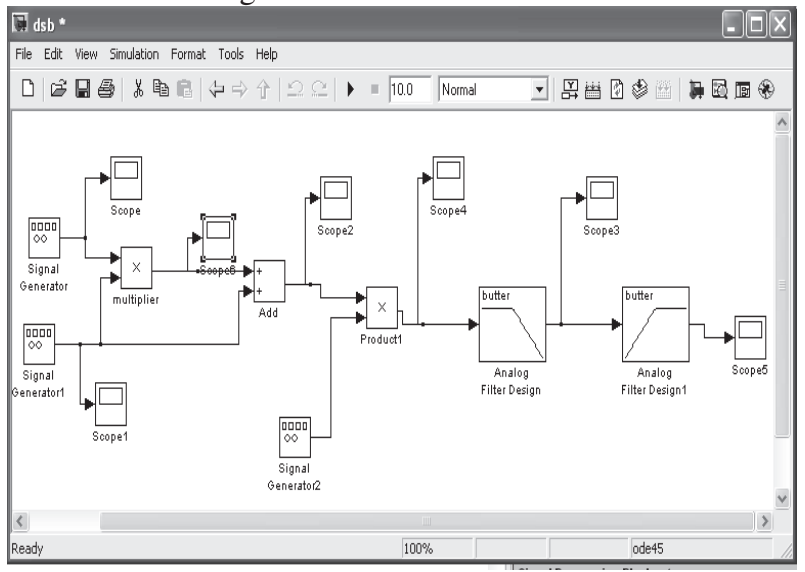

Fig.2. Simulink Model of Amplitude Modulation and demodulation

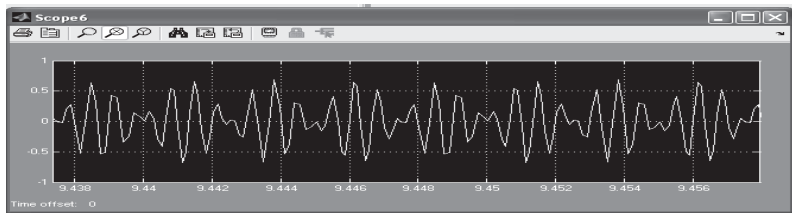

Fig.3. Simulation results of modulated signal.

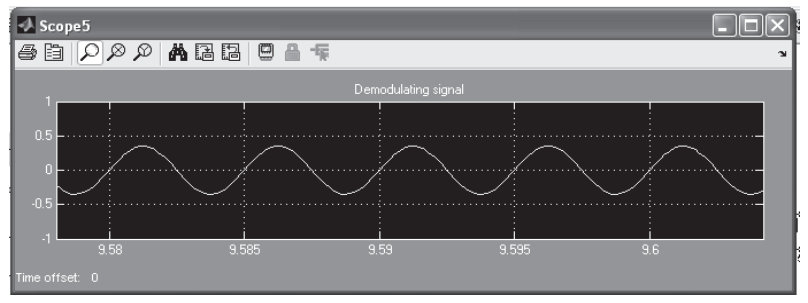

Fig.4. De-modulating Signal

B. Frequency Modulation: Frequency Modulation can be defined as Instantaneous carrier wave frequency is varied in accordance with the amplitude of the modulating or message signal. Keeping phase and amplitude is constant.

The FM signal is expressed as Equation (2).

$s(t)=A_{c}\left[\cos 2 \pi / f^{t}+\beta \sin (2 \pi / m)\right]$

Where Ac is the amplitude of the carrier signal, fc is the carrier frequency fm is the modulation index of the
FM wave. Depending on the value of $\beta$, FM can be either narrowband or wideband. For a small value of $\beta$ $(\beta<1)$ FM is known as NBFM (Narrow Band Frequency Modulation)and for a large value of $\beta$ ( $\beta>$ 1 ), it is known as WBFM(Wide Band Frequency Modulation). The Simulink model of Frequency modulation is shown below Fig.5.This model is designed for using the mathematical expression similar to Mat lab. Detector in this model is the slope detection principle. In this detector all the frequency variations are converted into the amplitude variations applied to the envelope detector. The simulation model of modulated signal is shown in Fig.6

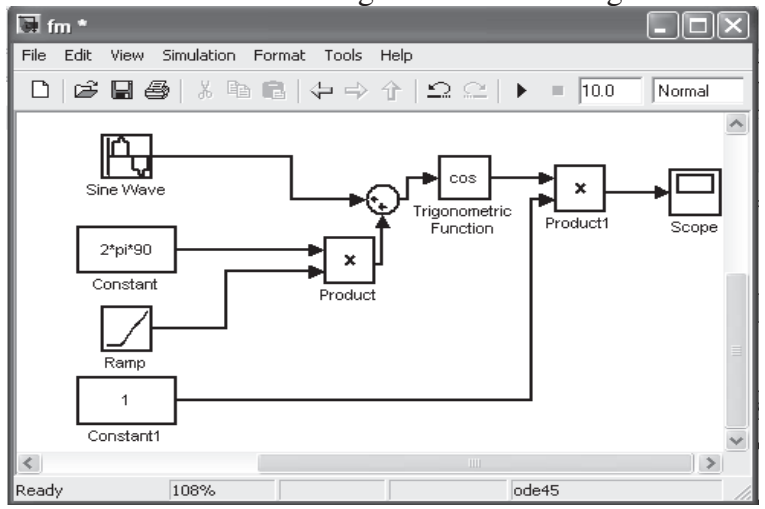

Fig.5. Simulink Model of Frequency Modulation

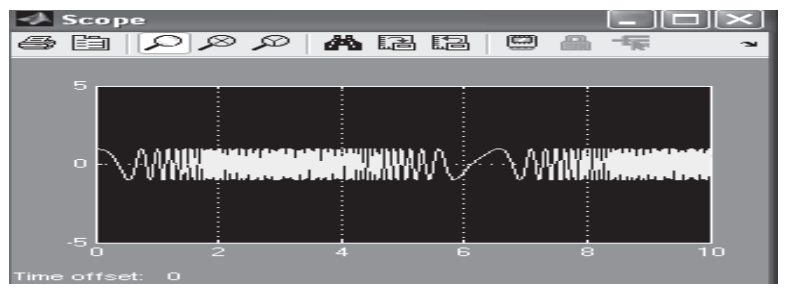

Fig.6. Simulation results of frequency modulated signal

\section{Conclusion}

MATLAB/Simulink is a very powerful tool that can be used for simulation in communication, control, DSP, etc. This paper builds a simple simulation model to illustrate the AM and FM techniques, how the communication block set of the Simulink allow you to implement it. AM and FM can be used not only for analog modulation but also for a platform to design other modulation system.

The advantage of Simulink model is not only for designing the communication system but also supports the hardware platform.

\section{REFERENCES}

[1] S. Haykin, Communication Systems, 4th Edition. Toronto: John Wiley \& Sons, Inc., 2001.

[2] Chowning, John; Bristow, David (1986). FM Theory \& Applications - By Musicians For Musicians. Tokyo: Yamaha. ISBN 4-636-17482-8. 
[3] Chowning, J. (1973). "The Synthesis of Complex Audio Spectra by Means of Frequency Modulation". Journal of the Audio Engineering Society 21 (7).

[4] Introduction to Matlab/Simulink for Engineers and Scientist 2nd Ed. By Ogbonnaya I. Okoro

[5] Goldsman, D, Nance, R. E., and Wilson, J. R., 2010, “A Brief History of Simulation Revisited," Proceedings of the 2010 Winter Simulation Conference, ed., B. Johansson, S. Jain, J. MontoyaTorres, J. Hugan, and E. Yücesan, pp. 567574, Piscataway, N.J.: Institute of Electrical and Electronics Engineers

[6] Ruth John (2003), Innovation and the Communications Revolution: From the Victorian Pioneers to Broadband Internet. Inst. of Electrical Engineers. pp. 59, 61-62. ISBN 0852962185

[7] The Math Works Inc., Simulink, Dynamic System Simulation for MATLAB, 3rd ed., 1999

[8] Okoro O. I, Analysis and Computer simulation of Electrical/Electronics Problems M.sc. lecture, 2014

[9] Gaines,1979, Scientific modelling basics. Technical Publications. p. 36. ISBN 978-81-8431-089- 4

[10] Mikael Olofsson, Signal Theory, 1st ed.: Holmbergs i Malmö AB, Sweden, 2011.

[11] Mao-Ching Chiu, Jung-Shan Lin Richard Chia Tung Lee, Communications Engineering: Essentials for Computer Scientists and Electrical Engineers, 1st ed., 2007.

[12] Duane Hanselman, Mastering MATLAB 7: Pearson Education International, 2005.

[13] Oborkhale Lawrence and Iroegbu Chibuisi,"

Modelling and Simulation of Frequency Modulated Radio Channel Using Matlab and Simulink" Journal of Multidisciplinary Engineering Science and Technology (JMEST) ISSN: 3159-0040 Vol. 2 Issue 4, April - 2015 